\title{
The Glueball Spectrum from Constituent Gluon Models
}

\section{Mathieu*†}

Université de Mons-Hainaut, Académie universitaire Wallonie-Bruxelles,

20 Place du Parc, B-7000 Mons, Belgique

E-mail: vincent.mathieu@umh.ac.be

\section{F. Buisseret}

Université de Mons-Hainaut, Académie universitaire Wallonie-Bruxelles,

20 Place du Parc, B-7000 Mons, Belgique

E-mail: fabien.buissereteumh.ac.be

\section{Semay ${ }^{\S}$}

Université de Mons-Hainaut, Académie universitaire Wallonie-Bruxelles, 20 Place du Parc, B-7000 Mons, Belgique

E-mail: claude.semay@umh.ac.be

\section{B. Silvestre-Brac}

LPSC Université Joseph Fourier, Grenoble 1

CNRS/IN2P3, Institut Polytechnique de Grenoble, 53 Avenue des Martyrs, F-38026

Grenoble-Cedex, France

E-mail: silvestredlpsc.in2p3.fr

We present a model for odd- $C$ (negative charge parity) glueballs with three constituent gluons. The model is an extension of a previous study of two-gluon glueballs. We show that, even if spin1 gluons seem to reproduce properly the lattice QCD spectrum for $C=+$ states, the extension for $C=-$ cannot match with the lattice results. Resorting to the helicity formalism, we show how transverse gluons fit in better agreement the lattice QCD spectrum. We then conclude that even if gluons gain an effective mass, they remain transverse particles.

8th Conference Quark Confinement and the Hadron Spectrum

September 1-6 2008

Mainz, Germany

\footnotetext{
* Speaker.

$\dagger$ IISN research worker.

F.R.S.-FNRS post-doctoral researcher.

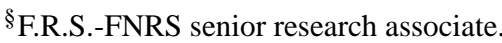




\section{Introduction}

Quantum Chromodynamics (QCD) allows the self-coupling of the gauge bosons, the gluons. Therefore, states with no valence quarks, the glueballs, are a beautiful consequence and prediction of QCD. Recently, a comprehensive review was devoted to the physics of glueballs [1].

Their observation, however, remains difficult, probably because the lightest glueball, the scalar $0^{++}$, should mix with mesons [2]. Some experimental glueball candidates are currently known, such as the $f_{0}(1370), f_{0}(1500), f_{0}(1710), \ldots$ but no definitive conclusions can be drawn concerning the nature of these states [1, 2].

On the other hand, pure gauge QCD has been investigated by lattice QCD for many years, leading to a well established glueball spectrum below $4 \mathrm{GeV}[3,4,5]$. Our aim is to reproduce this hierarchy with the most simple models with constituent gluons. Since two gluons can only bind into positive- $C$, we have to consider three-gluon glueballs to account for the existence of low-lying negative- $C$ states.

\section{Constituent models for two-gluon glueballs}

In ref. [6], the authors provide a relevant model of two-gluon glueballs. Assuming Casimir scaling for the string tension of the flux tube, the Hamiltonian, endowed with one-gluon exchange (OGE) potentials, reads

$$
H_{g g}=2 \sqrt{\boldsymbol{p}^{2}+m^{2}}+\frac{9}{4} \sigma r+V_{\text {oge }}\left(r ; \alpha_{S}, \mu ; \boldsymbol{S}, \boldsymbol{L}\right) .
$$

Although they use a bare mass $m=0$ in the kinetic term, their gluons have longitudinal components and are spin-1 particles. Therefore, many states are degenerate and the authors resorted to spindependent potentials coming from the OGE to lift these degeneracies. The corrections are of order $\mu^{-2}$, where $\mu=\left\langle\boldsymbol{p}^{2}\right\rangle$ is an effective constituent mass. The parameters were fitted on the low-lying states and the final spectrum is displayed in Fig. 1 (left).

All states (squares) fall into lattice error bars. However, we noticed some spurious states (circles) not found by any lattice study. For instance, $J=1$ states are forbidden by Yang's theorem and should not be present. The appearance of such states is induced by the longitudinal component of gluons and should disappear when considering transverse gluons.

\section{Odd- $C$ glueballs}

Let us forget about the spurious states for the moment and let us generalize the model of the previous section for three-gluon glueballs. We used a generalisation of the flux tube for the confinement. In heavy baryons, the confinement has a Y-shape, but in our case, we replaced it by a center-of-mass junction. The Hamiltonian is supplemented by the potential coming from the OGE and reads

$$
H_{g g g}=\sum_{i}^{3} \sqrt{\boldsymbol{p}_{i}^{2}}+\frac{9}{4} f \sigma\left|\boldsymbol{r}_{i}-\boldsymbol{R}_{c m}\right|+\sum_{i<j} V_{o g e}\left(r_{i j} ; \alpha_{S}, \mu ; \boldsymbol{L}_{i j}, \boldsymbol{S}_{i j}\right) .
$$

We refer the reader to the ref. [7] for further details concerning the Hamiltonian. This semirelativistic Hamiltonian is easily diagonalized in a Gaussian basis [8, 9] 
We impose the symmetric colour function $d_{a b c} A_{\mu}^{a} A_{v}^{b} A_{\rho}^{c}$, which ensures a negative $C$-parity. Then the spin symmetry determines the symmetry of the space. Since the coupling of three spin- 1 is given by

$$
\mathbf{1} \otimes \mathbf{1} \otimes \mathbf{1}=\mathbf{3}_{s} \oplus \mathbf{2}_{m} \oplus \mathbf{1}_{s} \oplus \mathbf{0}_{a},
$$

the lowest state with $2^{--}$has a mixed symmetry and cannot lie in the same mass range as $1^{--}$and $3^{--}$, as it was already noticed in ref. [10]. Moreover, a positive parity requires an odd angular momentum. Then, all $(0,1,2,3)^{+-}$are degenerate with a large component $L=1$ in the wave function. But the lattice QCD exhibits a gap around $2 \mathrm{GeV}$ between $0^{+-}$and $1^{+-}$which are respectively the highest and lowest states with a negative conjugation charge. This gap cannot be reproduced within this model and the spectrum of the Hamiltonian (3.1), shown in Fig. 1(right), is nearly in complete disagreement with lattice QCD. The symmetry arguments are Hamiltonian-independent and we can therefore conclude that models with longitudinal gluons are not appropriate to reproduce the lattice pure gauge spectrum.
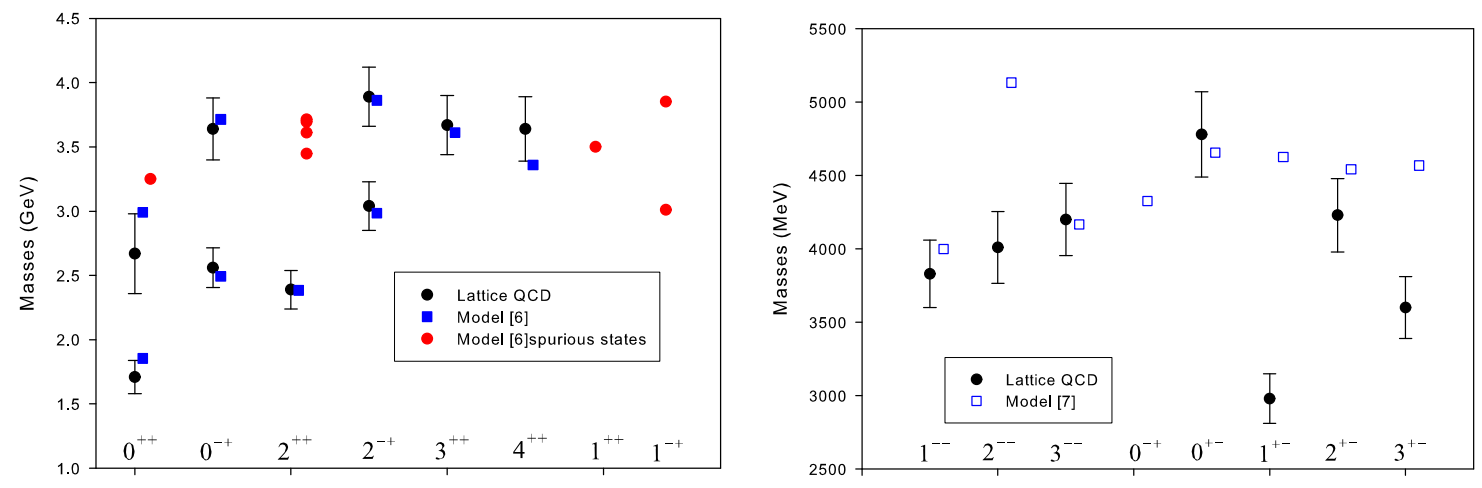

Figure 1: Left, spectrum of Hamiltonian (2.1) with longitudinal gluons; right, spectrum of Hamiltonian (3.1) with longitudinal gluons.

\section{Transverse gluons}

In order to solve the problems encountered (spurious states, hierarchy in the $P C=+-$ sector), we implemented a formalism developed by Jacob and Wick [11]. This formalism allows us to handle transverse particles. Indeed, in the previous models, longitudinal components implied extra states. When applying it to two-gluon glueballs, we remarked that the Bose symmetry (and the parity) implies selection rules. Three families were identified [12]: $(2 k)^{++},(2 k+3)^{++},(2 k+2)^{-+}$ with $k \in N$. One easily checks that no spurious $J=1$ states appear. Moreover, with this special construction, the orbital-spin part of all states is now expressed through a given linear combination of spectroscopic states. One does not need to use complicated spin-dependent potentials.

We checked the wave functions using a simple Hamiltonian:

$$
H_{g g}=2 \sqrt{p^{2}}+\frac{9}{4} \sigma r-3 \frac{\alpha_{S}}{r} .
$$




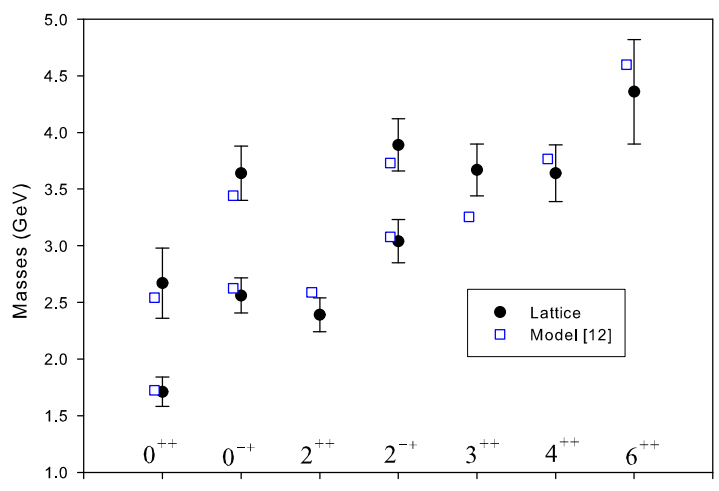

Figure 2: Spectrum of Hamiltonian (4.1) with transverse gluons.

The resulting spectrum, displayed in Fig. 2 is in good agreement with the lattice QCD data without the inclusion of spin-dependent potentials. But instanton-induced interactions were needed for $J=0$ states. In addition, all states are present with no spurious state. We then conclude that, in order to reproduce the glueball hierarchy observed in lattice QCD, one has to enforce that the lightest states with a positive $C$-parity are bound states of two transverse gluons.

The next step is to implement this formalism for three-gluon glueballs. This work is in progress. However, we have some indications that the lowest-lying odd- $C$ glueballs with transverse gluons are spin 1 and 3 [13]. Symmetry arguments are also in favour of a four-gluon interpretation for $0^{+-}$.

\section{References}

[1] V. Mathieu, N. Kochelev and V. Vento, The Physics of Glueballs, arXiv:0810.4453 [hep-ph].

[2] E. Klempt and A. Zaitsev, Glueballs, Hybrids, Multiquarks. Experimental facts versus QCD inspired concepts, Phys. Rept. 454, 1 (2007) [arXiv:0708.4016 [hep-ph] ] .

[3] C. J. Morningstar and M. Peardon, The Glueball spectrum from an anisotropic lattice study, Phys. Rev. D 60, 034509 (1999) [arXiv: hep-lat/9901004] .

[4] Y. Chen et al., Glueball spectrum and matrix elements on anisotropic lattices, Phys. Rev. D 73, 014516 (2006) [arXiv: hep-lat/0510074].

[5] H. B. Meyer and M. J. Teper, Glueball Regge trajectories and the pomeron: A lattice study, Phys. Lett. B 605, 344 (2005) [arXiv: hep-ph/0409183].

[6] F. Brau and C. Semay, Semirelativistic potential model for glueball states, Phys. Rev. D 70, 014017 (2004) [arXiv: hep-ph/0412173].

[7] V. Mathieu, C. Semay and B. Silvestre-Brac, Semirelativistic potential model for three-gluon glueballs, Phys. Rev. D 77, 094009 (2008) [arXiv:0803.0815 [hep-ph] ] .

[8] B. Silvestre-Brac and V. Mathieu, The few-body Problem in Terms of Correlated Gaussians, Phys. Rev. E 76 (2007) 046702 [arXiv: 0706.2300 [hep-ph] ] . 
[9] B. Silvestre-Brac and V. Mathieu, Spin Dependent Operators in Correlated Gaussians Basis, Phys. Rev. E 77, 036706 (2008) [arXiv:0712.0673 [physics.comp-ph] ] .

[10] V. Mathieu, C. Semay and B. Silvestre-Brac, Semirelativistic potential model for low-lying three-gluon glueballs, Phys. Rev. D 74, 054002 (2006) [arXiv : hep-ph/0605205] .

[11] M. Jacob and G. C. Wick, On the general theory of collisions for particles with spin, Ann. Phys. 7, 404 (1959).

[12] V. Mathieu, F. Buisseret and C. Semay, Gluons in glueballs: Spin or helicity? Phys. Rev. D 77, 114022 (2008) [arXiv:0802.0088 [hep-ph] ].

[13] N. Boulanger, F. Buisseret, V. Mathieu and C. Semay, Constituent gluon interpretation of glueballs and gluelumps, accepted for publication in Eur. J. Phys. A, arXiv:0806.3174 [hep-ph ] 